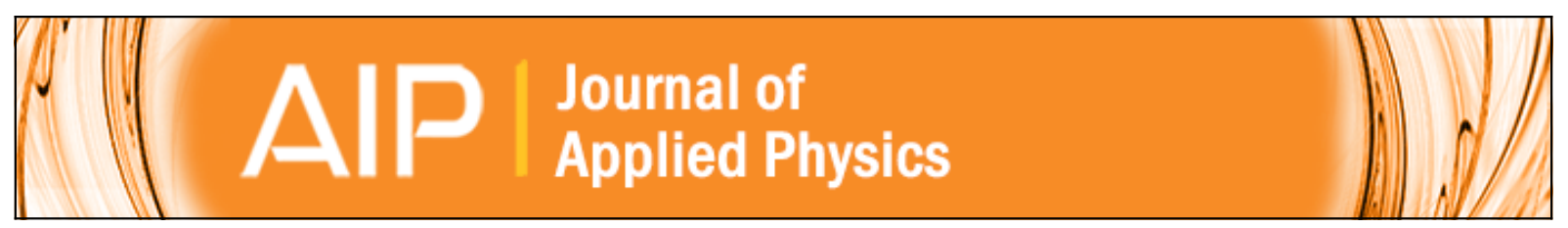

\title{
Transversal magnetic anisotropy in nanoscale PdNi-strips
}

D. Steininger, A. K. Hüttel, M. Ziola, M. Kiessling, M. Sperl, G. Bayreuther, and Ch. Strunk

Citation: Journal of Applied Physics 113, 034303 (2013); doi: 10.1063/1.4775799

View online: http://dx.doi.org/10.1063/1.4775799

View Table of Contents: http://scitation.aip.org/content/aip/journal/jap/113/3?ver=pdfcov

Published by the AIP Publishing

\section{Articles you may be interested in}

Large four-fold magnetic anisotropy in two-dimensional modulated Ni80Fe20 films

Appl. Phys. Lett. 107, 232402 (2015); 10.1063/1.4936994

Edge-modulated perpendicular magnetic anisotropy in [Co/Pd] $\mathrm{n}$ and $\mathrm{L} 10-\mathrm{FePt}$ thin film wires

Appl. Phys. Lett. 107, 182408 (2015); 10.1063/1.4935104

Magnetic-field-induced switchable exchange bias in NiFe film on (110) Fe3O4 with a strong uniaxial magnetic anisotropy

Appl. Phys. Lett. 106, 202405 (2015); 10.1063/1.4921487

Magnetic vortex crystal formation in the antidot complement of square artificial spin ice

Appl. Phys. Lett. 104, 092402 (2014); 10.1063/1.4867530

Thermomagnetic hysteresis effects in NiMn and NiMnPd thin films

J. Appl. Phys. 89, 7044 (2001); 10.1063/1.1362650

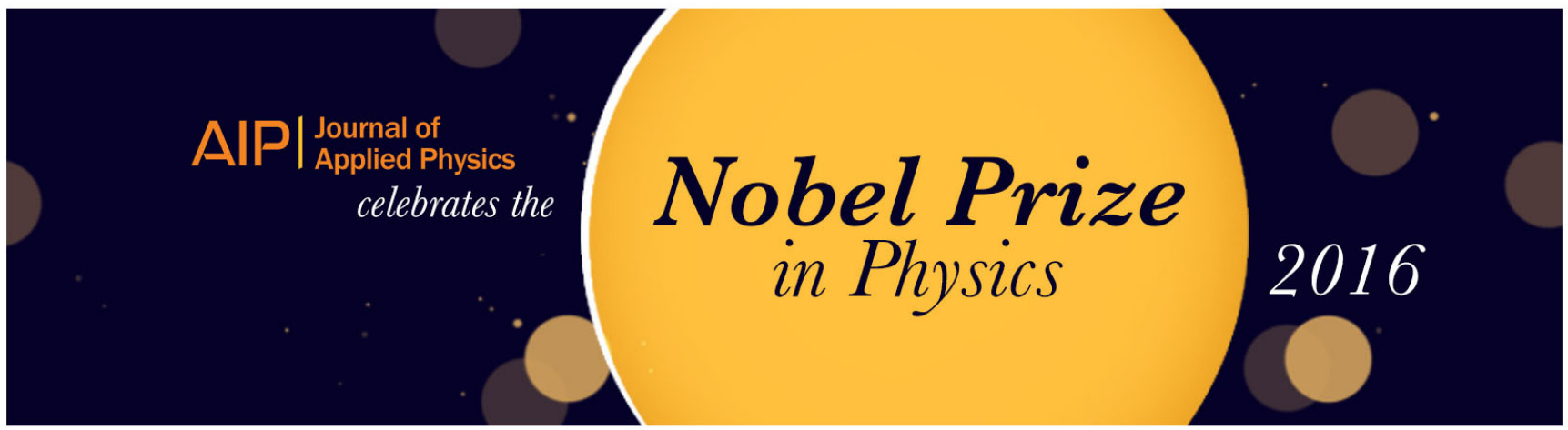




\title{
Transversal magnetic anisotropy in nanoscale PdNi-strips
}

\author{
D. Steininger, ${ }^{\text {a) }}$ A. K. Hüttel, M. Ziola, M. Kiessling, M. Sperl, G. Bayreuther, and Ch. Strunk \\ Institute for Experimental and Applied Physics, University of Regensburg, Universitätsstr. 31, \\ D-93053 Regensburg, Germany
}

(Received 17 October 2012; accepted 26 December 2012; published online 15 January 2013)

\begin{abstract}
We investigate submicron ferromagnetic PdNi thin-film strips intended as contact electrodes for carbon nanotube-based spintronic devices. The magnetic anisotropy and micromagnetic structure are measured as function of temperature. Contrary to the expectation from shape anisotropy, magnetic hysteresis measurements of $\mathrm{Pd}_{0.3} \mathrm{Ni}_{0.7}$ on arrays containing strips of various width point towards a magnetically easy axis in the sample plane, but transversal to the strip direction. Anisotropic magnetoresistance measured on individual $\mathrm{Pd}_{0.3} \mathrm{Ni}_{0.7}$ contact strips and magnetic force microscopy images substantiate that conclusion. (C) 2013 American Institute of Physics. [http:// dx.doi.org/10.1063/1.4775799]
\end{abstract}

\section{INTRODUCTION}

Carbon nanotubes (CNTs) have become a frequently used material for spin dependent transport experiments within the past years. Due to spin lifetimes of several nanoseconds, ${ }^{1,2}$ CNTs provide excellent conditions for this kind of investigation. ${ }^{3-6}$ Spin injection requires ferromagnetic contact electrodes to the CNT which provide both spin polarization and a transparent electric contact. As palladium is known for its high contact transparency to CNTs, ${ }^{3,5,7-9}$ an alloy of palladium with nickel as ferromagnetic material was chosen for this study. The electronic properties of pure palladium $^{10,11}$ and especially the formation of giant magnetic moments in dilute alloys with small amounts of ferromagnetic elements have been well studied for both bulk material and thin films, with respect to Curie points and critical alloy concentrations for the onset of ferromagnetism. ${ }^{12-17}$

Objective of this work is the investigation of thin film $\mathrm{Pd}_{0.3} \mathrm{Ni}_{0.7}$ high aspect ratio shapes, particularly with regard to their suitability as ferromagnetic contact electrodes for CNT devices. While the similar alloy $\mathrm{Pd}_{1-x} \mathrm{Fe}_{x}$ is governed by shape anisotropy, $4,12,13,18,19$ this finding is not transferable to $\mathrm{Pd}_{1-x} \mathrm{Ni}_{x}$ due to the significantly different crystal structures of nickel and iron and the differing ferromagnet concentrations in the experiments. ${ }^{20}$

We use $\mathrm{Pd}_{0.3} \mathrm{Ni}_{0.7}$ strips at widths of $250-1500 \mathrm{~nm}$, maintaining a constant length of $5 \mu \mathrm{m}$ and metal film thickness of $50 \mathrm{~nm}$. All samples were fabricated on a boron doped, $\mathrm{p}++$ type $\mathrm{Si}$ substrate with thermally grown $\mathrm{SiO}_{2}$ surface oxide, and structured via electron beam lithography and lift-off process using a polymethyl methacrylate two layer resist. The deposition of the thin films was done by electron beam evaporation from pre-mixed bulk material in an UHV evaporation system with a base pressure of $4 \times 10^{-8}$ mbar. Figure 1 displays such a strip pattern, in this particular case with leads attached in a second lithography step for magnetoresistance measurements. It also introduces the characteristic directions for all the magnetic field orientations used in this manuscript, i.e., perpendicular $(\mathrm{P})$,

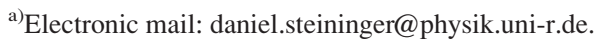

transverse $(\mathrm{T})$, and longitudinal (L) with respect to the $\mathrm{Pd}_{0.3} \mathrm{Ni}_{0.7}$ strip.

\section{SQUID MAGNETIZATION MEASUREMENTS}

Superconducting quantum interference device (SQUID) magnetization measurements using a commercial magnetometer have been used to characterize the averaged magnetic hysteresis of arrays containing $1.3 \times 10^{6}$ non-contacted, electrically isolated thin film strips. They reveal the switching characteristics at various temperatures and orientations of the external magnetic field.

Fig. 2 displays the magnetization curves of an array of $250 \mathrm{~nm}$ wide strips for two different temperatures after subtraction of the diamagnetic and paramagnetic moments of the boron doped Si substrate, the latter showing a strongly field-dependent susceptibility at low temperature which was fitted with a Brillouin function. The total magnetic moment of the sample at $\mathrm{T}=300 \mathrm{~K}$ is $M_{\text {tot }}=3.7 \times 10^{-5} \mathrm{Gcm}^{3}$ (see Fig. 2(a)), from which an average magnetic moment of $\bar{\mu}=0.583 \mu_{B}$ per alloy atom can be calculated. This value is

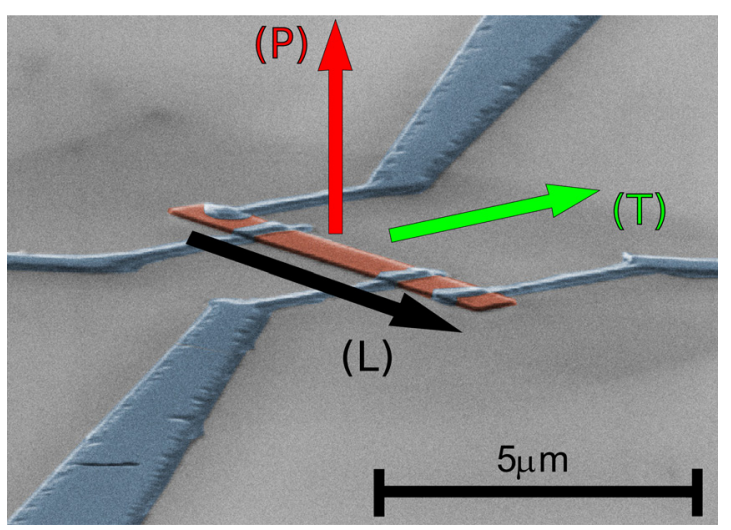

FIG. 1. Scanning electron microscopy (SEM) image of a contacted $\mathrm{Pd}_{0.3} \mathrm{Ni}_{0.7}$ strip (colorized in red). The four contact leads (colorized blue) were evaporated in a second step from pure palladium. They provide the possibility to perform four terminal resistance measurements. The arrows indicate the three tested orientations of the external magnetic field relative to the strip, namely black $(\mathrm{L})$ : longitudinal, red $(\mathrm{P})$ : perpendicular, and green (T): transverse. 

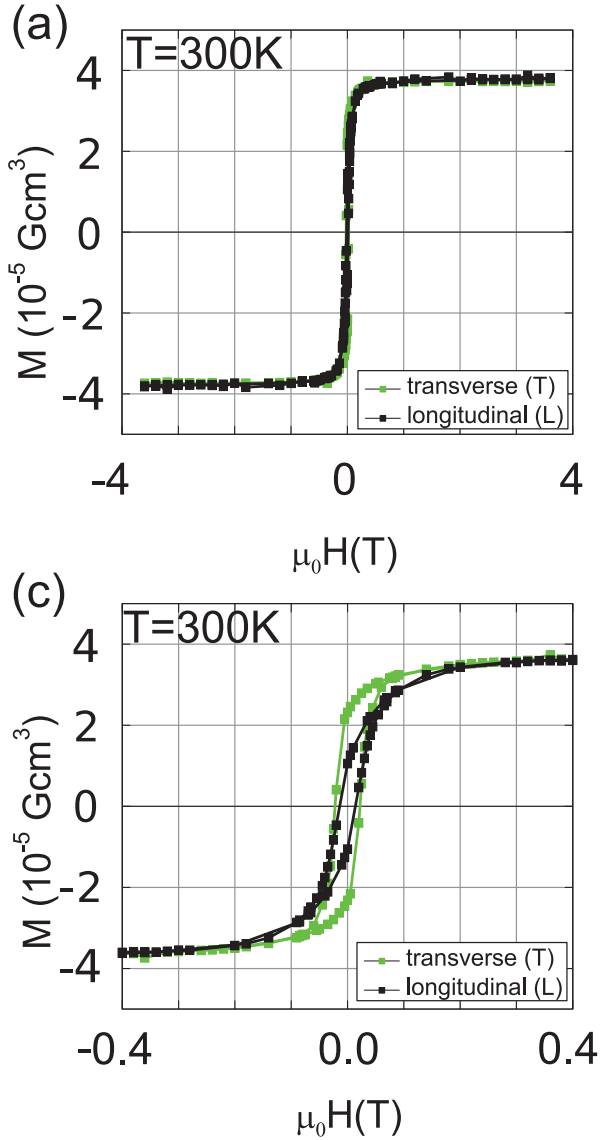

(b)

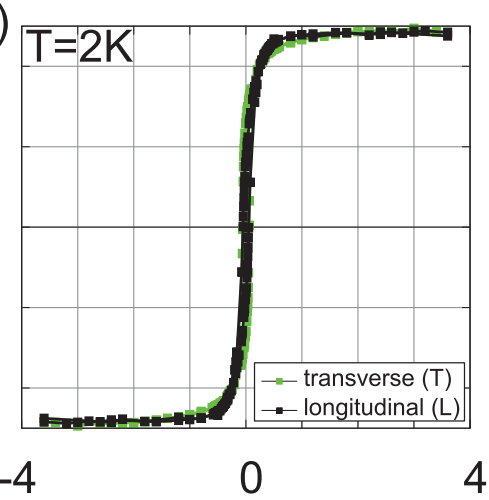

(d)

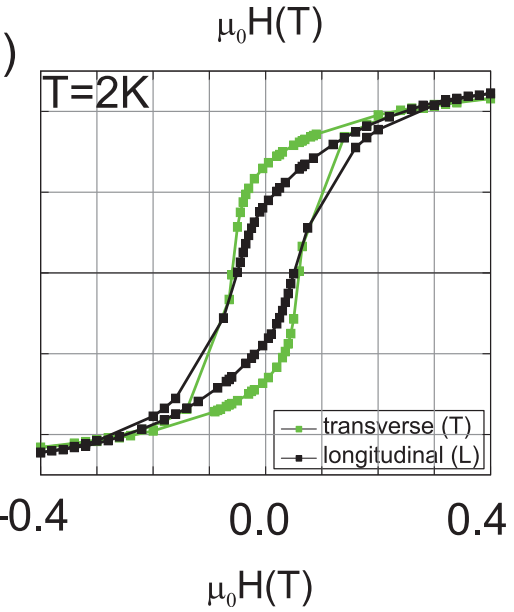

FIG. 2. SQUID magnetization measurements on an array containing $1.3 \times 10^{6}$ strips of $250 \mathrm{~nm}$ width and $5 \mu \mathrm{m}$ length. The array was measured in an external field of $\mu_{0}|H| \leq 3.6 \mathrm{~T}$ in both transverse $(\mathrm{T}$, green) and longitudinal $(\mathrm{L}$, black $)$ direction (see also Fig. 1) at $T=300 \mathrm{~K}(\mathrm{a}, \mathrm{c})$ and $T=2 \mathrm{~K}(\mathrm{~b}, \mathrm{~d})$. The upper panels $(\mathrm{a}, \mathrm{b})$ show the full field range of the hysteresis loop while the lower panels (c,d) zoom into the range $-0.4 \mathrm{~T} \leq \mu_{0} H \leq 0.4 \mathrm{~T}$. comparable to previous results for bulk $\mathrm{Pd}_{0.3} \mathrm{Ni}_{0.7}$, where also the Curie temperature of this material has been characterized $\left(T_{C} \approx 532 \mathrm{~K}\right) .^{12,13}$ Figures $2(\mathrm{a})$ and $2(\mathrm{~b})$ show magnetization loops in a transverse $(\mathrm{T})$ and longitudinal $(\mathrm{L})$ external field at $T=300 \mathrm{~K}$ and $T=2 \mathrm{~K}$, while Figures 2 (c) and $2(\mathrm{~d})$ zoom in onto the range $-0.4 \mathrm{~T} \leq \mu_{0} H \leq 0.4 \mathrm{~T}$. The higher field required for saturation and the larger coercive field values observed at low temperature can be explained by the absence of thermally activated motion of domain walls. Saturation is reached at lower external fields for the transverse orientation of external magnetic field. Also the remanent magnetization in transverse direction is significantly higher than in longitudinal orientation. These observations indicate an initially unexpected magnetic anisotropy with the easy axis along the transverse direction (T).

Concerning the details of the magnetization curves, the remanent magnetization in (T) direction is not $100 \%$ as expected for an easy axis. This can be explained by the strong demagnetizing field connected with the magnetization pointing perpendicular to the edge of the sample. This is expected to cause some reversed domains as indicated by magnetic force microscopy (MFM) images discussed below (Fig. 4(b)). Furthermore, to avoid a large related magnetostatic energy, the magnetization will rotate towards the $(\mathrm{L})$ direction close to the lateral edges. Hence, the resulting average transverse magnetization component decreases with decreasing strip width and becomes substantially reduced for sub-micrometer dimensions. On the other hand, the remanence in the (L) direction is not zero as would be expected for a uniaxial hard axis. This is presumably a result of the domain splitting in the remanent state after saturation along the hard axis, which is confirmed by the MFM images shown in Fig. 4(a) (see below). For small domains with a width below $300 \mathrm{~nm}$ as deduced from Fig. 4(a), the magnetization inside and in the vicinity of the Néel type domain walls will have a substantial component perpendicular to the wall, i.e., along the (L) direction, which shows up as the longitudinal remanence.

\section{MAGNETOTRANSPORT}

To obtain further information on the direction of spontaneous magnetization and the switching behaviour of single contact electrodes, the anisotropic magnetoresistance (AMR) of individually contacted $\mathrm{Pd}_{0.3} \mathrm{Ni}_{0.7}$ strips was measured. As with the samples for the SQUID measurements, the strips were fabricated via electron beam lithography and lift-off process on identical $\mathrm{Si} / \mathrm{SiO}_{2}$ substrate material. The nonmagnetic palladium leads (see Fig. 1) were deposited using a second electron beam lithography and evaporation step. The resistance of a ferromagnetic strip is generally higher when the current through the strip is aligned parallel to the magnetization vector of the structure $(\vec{J} \| \vec{M})$ than in the perpendicular case $(\vec{J} \perp \vec{M}) .^{21-24}$ Transport measurements were performed at $T=4.2 \mathrm{~K}$, applying a maximum external magnetic field of $\mu_{0} H_{\max }= \pm 925 \mathrm{mT}$ successively in different orientations relative to the strip. The resistance measurement itself was carried out using a four-terminal setup and lock-in technique.

Fig. 3 shows the relative change of resistance $\Delta R$ $=\left(R\left(\mu_{0} H\right)-R_{\max }\right) / R_{\max }$, in dependence on the external 


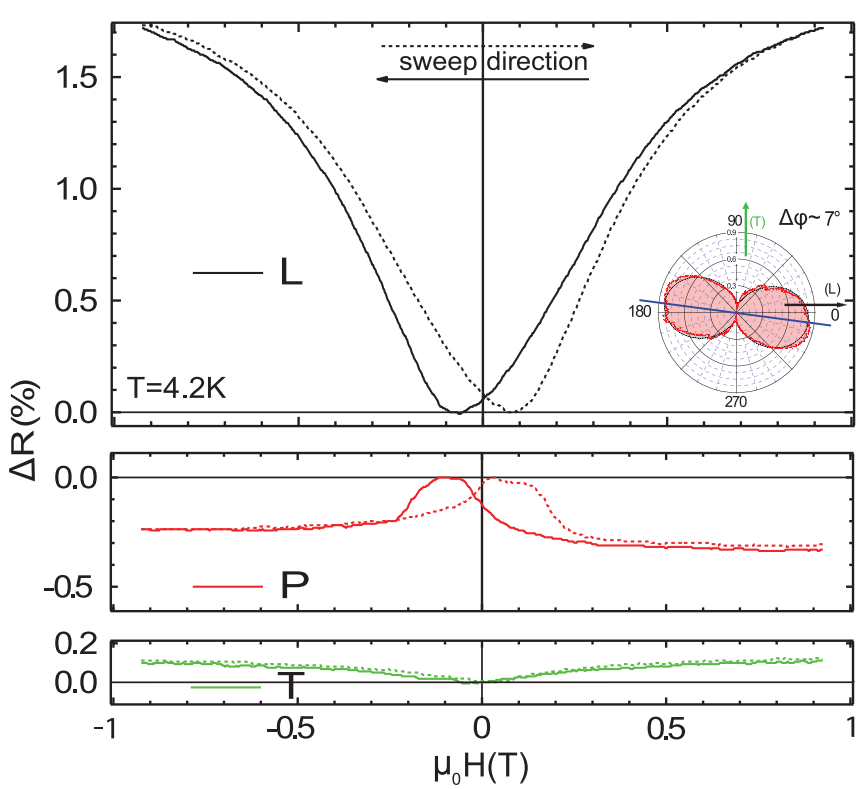

FIG. 3. Anisotropic magnetoresistance $\Delta R=\left(R\left(\mu_{0} H\right)-R_{\max }\right) / R_{\max }$ of an individually contacted, $500 \mathrm{~nm}$ wide $\mathrm{Pd}_{0.3} \mathrm{Ni}_{0.7}$ strip. The three panels represent the relative orientiation of the magnetic field according to Fig. 1, i.e., longitudinal (L, black), perpendicular $(\mathrm{P}, \mathrm{red})$ and transverse $(\mathrm{T}$, green). The dashed traces show the magnetic field up-sweep, while the continuous traces show the down-sweep measurement. All three panels have identical scaling of both axes. The inset shows a polar plot of the AMR at constant magnetic field $\left|\mu_{0} H\right|=1 \mathrm{~T}$ and reveals a small misalignment of $\Delta \phi \approx 7^{\circ}$.

magnetic field $\mu_{0} H$, for a strip width of $500 \mathrm{~nm}$. The three panels correspond to the three field orientations as sketched in Fig. 1. The largest increase of resistance occurs when applying the field in longitudinal (L) direction. This means that a large part of the magnetization was oriented away from this direction and had to be aligned parallel to the strip by the external magnetic field. In perpendicular $(\mathrm{P})$ direction, we observe a decrease of the resistance with increasing perpendicular field, i.e., with the alignment of the magnetization perpendicular to the film plane and the in-plane current. This means that the magnetization must have a longitudinal component in the remanent state. In transverse (T) direction, a small but finite increase of resistance appears with increasing field. This is explained by a misalignment of the external
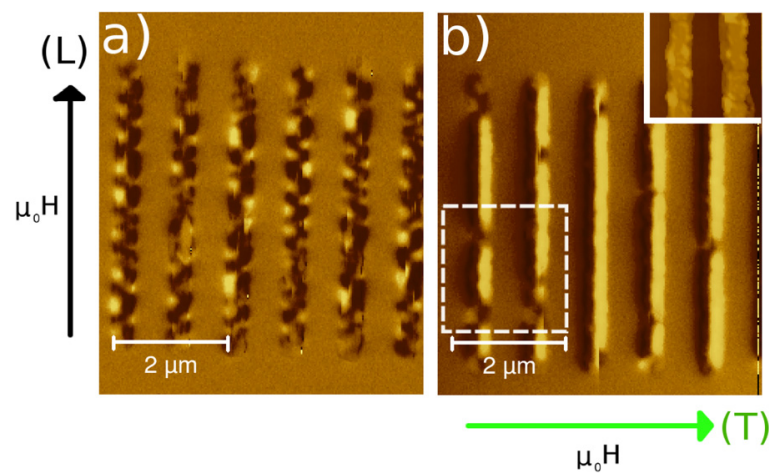

FIG. 4. Magnetic force microscopy (MFM) images of the remanent magnetization of an ensemble of $500 \mathrm{~nm}$ wide $\mathrm{Pd}_{0.3} \mathrm{Ni}_{0.7}$ strips. Before recording the image, the strips have been magnetized along the directions indicated by the arrows, i.e., (a) in longitudinal (L), and (b) in transverse (T) direction. The inset in (b) shows the surface topography of the region in the white dashed square. field by $7^{\circ}$ relative to the (T) axis which was verified by measuring the angular variation of the magnetoresistance in a constant field of $\mu_{0} H=1 \mathrm{~T}$ (see inset of Fig. 3). A consistent interpretation of all three magnetoresistance curves in agreement with the SQUID magnetization curves is obtained by assuming that after saturation along a hard axis, i.e., either along an $(\mathrm{L})$ or a $(\mathrm{P})$ direction the magnetization is split into domains, as seen in Fig. 4(a), with a small M component along (L) due to Néel type domain walls and close to the long edge of the strip. The presence of domain walls in the remanent state is also responsible for the very similar hysteretic behaviour of the magnetization loops (Fig. 2) and the magnetoresistance curves (Fig. 3) along the (L) axis.

The distinct reduction of the remanence along the $\mathrm{T}$ axis below $100 \%$ due to the formation of some reversed domains (see Fig. 4(b)) has practically no effect on the magnetoresistance because the AMR scales with $\delta R \propto \cos ^{2}(\alpha)$ while the magnetometer measures the parallel component of the average magnetization $\mathrm{M}_{\|} \propto \cos (\alpha)$, where $\alpha$ is the angle between the (T) axis and the domain magnetization.

As a result, we can conjecture that the spontaneous magnetization of the strips is oriented mainly transverse to the strips' long axis. The small amount of change in resistance in a magnetic field along the transverse (T) direction means that the majority of magnetic moments already was aligned in this direction before the field was applied. The minima, respectively, maxima of the magnetoresistance curves show hysteretic behaviour and are mirrored according to the sweep direction of the external field.

\section{MAGNETIC FORCE MICROSCOPY}

Finally, MFM on a strip array directly reveals the remanent micromagnetic configuration within narrow strips (width $500 \mathrm{~nm}$ ) of $\mathrm{Pd}_{0.3} \mathrm{Ni}_{0.7}$. Fig. 4 displays MFM images of an ensemble of contact strips. The MFM pictures were taken at room temperature and zero external magnetic field; the scanning direction was longitudinal to the strips (L). Prior to imaging, the sample was magnetized along the longitudinal (L) (Fig. 4(a)) or transverse (T) direction (Fig. 4(b)) by an external field of $\mu_{0} H=2 \mathrm{~T}$ to ensure magnetic saturation.

The pattern in Fig. 4(a), where the sample was previously magnetized in longitudinal (L) direction, features alternating domain alignment along the transverse $(\mathrm{T})$ direction. This is consistent with a spontaneous magnetization in transverse (T) axis, as inferred from the SQUID and AMR measurements. This splitting in small domains in the remanent state also causes the non-zero remanence in the (L) direction observed in the SQUID data (see Fig. 2) as explained before. In Fig. 4(b), after magnetizing in (T) direction the magnetization structure in this direction is considerably more uniform. The strips feature large domains with transverse (T) magnetization direction. The remaining disintegration into domains in certain areas may be caused by, e.g., edge roughness of the strips, supported by thermal activated processes at room temperature: in the inset of Fig. 4(b), showing the surface topography of the region marked by a white dashed square in the MFM picture, several magnetic features reappear as height profile irregularities. Altogether, MFM 
imaging again confirms the findings of an easy axis along the transverse $(\mathrm{T})$ direction.

\section{DISCUSSION}

A possible explanation for the transverse magnetic easy axis is the effect of inverse magnetostriction. ${ }^{25}$ While many mechanisms can contribute local stress during fabrication of the strips, it is already instructive to look at the highly different thermal expansion coefficients $\alpha$ of the thin film metal $\left(\alpha_{\mathrm{Pd}} \approx 11.8 \times 10^{-6} \mathrm{~K}^{-1}, \quad \alpha_{\mathrm{Ni}} \approx 13.4 \times 10^{-6} \mathrm{~K}^{-1}\right)$ and the substrate material used in this study $\left(\alpha_{\mathrm{Si}} \approx 2.6 \times 10^{-6} \mathrm{~K}^{-1}\right)$. Considering a temperature higher than room temperature during the thin film evaporation process of $\mathrm{PdNi}$, tensile stress is imprinted on the metal layer when the sample is cooled down. At the edges of the strips, this stress can relax. If the strip is sufficiently narrow, the relaxation of transversal stress takes place across the entire strip. Assuming a similar behaviour of $\mathrm{Pd}_{0.3} \mathrm{Ni}_{0.7}$ and pure nickel (due to the high amount of nickel in our alloy and the same crystal structure (fcc) of both elements), the magnetic moments in the strip then align orthogonal to the remaining longitudinal stress, i.e., in the transversal $(\mathrm{T})$ direction, as the magnetostriction coefficient of nickel is negative for both (100) and (111) direction. ${ }^{25}$

\section{CONCLUSIONS}

As a conclusion, our work confirms in all aspects that the magnetic preferential direction of high aspect ratio $\mathrm{Pd}_{0.3} \mathrm{Ni}_{0.7}$ thin film contact strips feature a magnetically easy axis transverse to the strip orientation. This can be explained by the effect of magneto-elastic coupling. It dominates other mechanisms as, e.g., shape anisotropy, which would favour a magnetically easy axis longitudinal to the strip orientation. For application of our investigated contact strips in CNT based spin devices, it is therefore recommended to apply external magnetic fields in transverse direction in order to obtain a more distinct switching behaviour. Our results are well compatible with those of the complementary study by Chaleau et al., ${ }^{20}$ who did extensive micromagnetic simulations and investigated the remanent magnetization direction using XMCD experiments.

\section{ACKNOWLEDGMENTS}

The authors would like to thank J.-Y. Chauleau for helpful discussions. We gratefully acknowledge funding by the EU FP7 project SE2ND and by the Deutsche Forschungsgemeinschaft via SFB 689, GRK 1570, and Emmy Noether project $\mathrm{Hu}$ 1808/1-1.

${ }^{1}$ K. Tsukagoshi, B. W. Alphenaar, and H. Ago, "Coherent transport of electron spin in a ferromagnetically contacted carbon nanotube," Nature 401, 572 (1999).
${ }^{2}$ L. E. Hueso, J. M. Pruneda, V. Ferrari, G. Burnell, J. P. Valdés-Herrera, B. D. Simons, P. B. Littlewood, E. Artacho, A. Fert, and N. D. Mathur, "Transformation of spin information into large electrical signals using carbon nanotubes." Nature 445, 410-413 (2007).

${ }^{3}$ S. Sahoo, T. Kontos, J. Furer, C. Hoffmann, M. Graber, A. Cottet, and C. Schönenberger, "Electric field control of spin transport," Nature Phys. 1, 99-102 (2005).

${ }^{4}$ D. Preusche, S. Schmidmeier, E. Pallecchi, C. Dietrich, A. K. Hüttel, J. Zweck, and C. Strunk, "Characterization of ferromagnetic contacts to carbon nanotubes," J. Appl. Phys. 106, 084314 (2009).

${ }^{5}$ M. Gaass, A. K. Hüttel, K. Kang, I. Weymann, J. von Delft, and C. Strunk, "Universality of the Kondo effect in quantum dots with ferromagnetic leads," Phys. Rev. Lett. 107, 176808 (2011).

${ }^{6}$ H. Aurich, A. Baumgartner, F. Freitag, A. Eichler, J. Trbovic, and C. Schönenberger, "Permalloy-based carbon nanotube spin-valve," Appl. Phys. Lett. 97, 153116 (2010).

${ }^{7}$ M. J. Biercuk, S. Garaj, N. Mason, J. M. Chow, and C. M. Marcus, "Gatedefined quantum dots on carbon nanotubes," Nano Lett. 5, 1267-1271 (2005).

${ }^{8}$ S. Sahoo, T. Kontos, C. Schönenberger, and C. Sürgers, "Electrical spin injection in multiwall carbon nanotubes with transparent ferromagnetic contacts," Appl. Phys. Lett. 86, 112109 (2005).

${ }^{9}$ C. Feuillet-Palma, T. Delattre, P. Morfin, J. M. Berroir, G. Fève, D. C. Glattli, B. Plaçais, A. Cottet, and T. Kontos, "Conserved spin and orbital phase along carbon nanotubes connected with multiple ferromagnetic contacts," Phys. Rev. B 81, 115414 (2010).

${ }^{10}$ F. M. Mueller, A. J. Freeman, J. O. Dimmock, and A. M. Furdyna, "Electronic structure of palladium," Phys. Rev. B 1, 4617-4635 (1970).

${ }^{11}$ L. Hodges, R. E. Watson, and H. Ehrenreich, "Renormalized atoms and the band theory of transition metals," Phys. Rev. B 5, 3953-3971 (1972).

${ }^{12}$ J. C. Ododo, "Percolation concentration and saturation of the Pd moment in ferromagnetic Pd alloys," J. Phys. F: Met. Phys. 13, 1291-1309 (1983).

${ }^{13} \mathrm{~J}$. C. Ododo, "The onset of ferromagnetism in transition metal alloys as a cooperative phase transition," J. Phys. F: Met. Phys. 10, 2515-2534 (1980).

${ }^{14}$ G. J. Nieuwenhuys, "Magnetic behaviour of cobalt, iron and manganese dissolved in palladium," Adv. Phys. 24, 515 (1975).

${ }^{15}$ E. O. Wollan, "Magnetic coupling in the Ni-Pd alloy system," Phys. Rev. 167, 461-463 (1968).

${ }^{16} \mathrm{~J}$. W. Loram and K. A. Mirza, "Dilute PdNi-a homogeneous magnetic system of fluctuating moments," J. Phys. F: Met. Phys. 15, 2213-2229 (1985).

${ }^{17}$ H. Z. Arham, T. S. Khaire, R. Loloee, W. P. Pratt, and N. O. Birge, "Measurement of spin memory lengths in PdNi and PdFe ferromagnetic alloys," Phys. Rev. B 80, 174515 (2009).

${ }^{18}$ S. Schmidmeier, "Mikromagnetische Charakterisierung von ferromagnetischen Kontaktelektroden,” Diploma Thesis (Universität Regensburg, 2007).

${ }^{19}$ J. C. Ododo, "Ferromagnetic correlation lengths in dilute PdFe and PdCo alloys,” J. Phys. F: Met. Phys. 15, 941-951 (1985).

${ }^{20}$ J.-Y. Chauleau, B. J. McMorran, R. Belkhou, N. Bergeard, T. O. Menteş, M. A. Niño, A. Locatelli, J. Unguris, S. Rohart, J. Miltat, and A. Thiaville, "Magnetization textures in NiPd nanostructures," Phys. Rev. B 84, 094416 (2011).

${ }^{21}$ While local orbital moments may lead to an exception from this rule specifically in PdNi at Ni concentrations below 50\%, this is not the case for the high Ni concentration present here (see Ref. 24 for details).

${ }^{22}$ T. McGuire and R. Potter, "Anisotropic magnetoresistance in ferromagnetic 3d alloys," IEEE Trans. Magn. 11, 1018-1038 (1975).

${ }^{23}$ J. C. Gonzalez-Pons, J. J. Henderson, E. del Barco, and B. Ozyilmaz, "Geometrical control of the magnetization direction in high aspect-ratio PdNi ferromagnetic nanoelectrodes," Phys. Rev. B 78, 012408 (2008).

${ }^{24}$ S. Senoussi, I. Campbell, and A. Fert, "Evidence for local orbital moments on Ni and Co impurities in Pd," Solid State Commun. 21, 269-271 (1977).

${ }^{25}$ S. Chikazumi, Physics of Ferromagnetism, 2nd ed. (Clarendon, Oxford, 1997). 